\title{
Pretransplant Locoregional Therapy for Hepatocellular Carcinoma: Evaluation of Explant Pathology and Overall Survival
}

\author{
Eliza W. Beal', Kristin M. Dittmar', A. James Hanje ${ }^{3}$, Anthony J. Michaels ${ }^{3}$, \\ Lanla Conteh ${ }^{3}$, Gail Davidson', Sylvester M. Black', P. Mark Bloomston', \\ Mary E. Dillhoff' and Carl R. Schmidt ${ }^{1 *}$
}

\begin{abstract}
'Department of Surgery, The Ohio State University Wexner Medical Center, James Cancer Hospital, Columbus, OH, USA, ${ }^{2}$ Department of Radiology, The Ohio State University Wexner Medical Center, James Cancer Hospital, Columbus, OH, USA, ${ }^{3}$ Division of Gastroenterology, Hepatology and Nutrition, Department of Internal Medicine, The Ohio State University Wexner Medical Center, Columbus, OH, USA
\end{abstract}

OPEN ACCESS

Edited by:

Nikolaos Arkadopoulos, Athens University School of Medicine,

Greece

Reviewed by: Kun-Ming Chan,

Chang Gung Memorial Hospital at

Linkou, Taiwan

Evangelos P. Misiakos,

National and Kapodistrian University

of Athens, Greece

${ }^{*}$ Correspondence:

Carl R. Schmidt

carl.schmidt@osumc.edu

Specialty section: This article was submitted to Surgical Oncology, a section of the journal

Frontiers in Oncology

Received: 05 April 2016 Accepted: 26 May 2016 Published: 13 June 2016

Citation:

Beal EW, Dittmar KM, Hanje AJ, Michaels AJ, Conteh L, Davidson G, Black SM, Bloomston PM, Dillhoff ME and Schmidt CR (2016) Pretransplant Locoregional Therapy for

Hepatocellular Carcinoma:

Evaluation of Explant Pathology and Overall Survival.

Front. Oncol. 6:143.

doi: 10.3389/fonc.2016.00143
Background and objectives: Liver transplant is an important treatment option for patients with hepatocellular carcinoma $(\mathrm{HCC})$ within Milan criteria. We sought to determine the rate of complete tumor necrosis after bridging therapy.

Methods: The medical records of all 178 patients undergoing liver transplantation between January 1, 2008 and July 31, 2015 were reviewed. Response to therapy by imaging was based on mRECIST criteria (1).

Results: Sixty-three (35\%) patients had HCC. Forty-three (68\%) were treated with at least one bridging therapy and 14 (22\%) were diagnosed incidentally. Eighteen (42\%) underwent TACE and 25 (58\%) underwent ablation. Twenty (80\%) patients who underwent ablation and nine (60\%) who underwent TACE had complete response based on imaging. Viable tumor was identified in explant pathology in 32 patients (74\%). The presence or absence of viable tumor was not associated with overall survival.

Conclusion: Rates of viable tumor based on pathologic analysis in the hepatic explant were high after bridging therapy, but not associated with worse outcome. We conclude that serial bridging to achieve complete pathologic tumor response is not needed prior to transplant for HCC, and presence of complete response by imaging is adequate. Further studies are needed to determine if cancer cells that appear viable are alive.

Keywords: liver transplantation, carcinoma, hepatocellular, chemoembolization, therapeutic, radiofrequency ablation, microwave ablation, locoregional bridging therapy

\section{INTRODUCTION}

Hepatocellular carcinoma (HCC) is one of the few cancers with increasing incidence in the United States over the past two decades (2-4). Orthotopic liver transplant is widely accepted as a potentially curative treatment option for patients with early stage cancer defined by the Milan criteria (5-8). One limitation of this therapy is the growing incidence of waitlist dropouts due to increasing time on the list related to limited organ availability (9). In a recent consensus statement published in the Journal 
of the National Comprehensive Cancer Network (JNCCN), it is recommended that patients with HCC be considered for bridging therapy (10) if they are expected to wait more than 6 months for transplantation in order to reduce dropouts from the waitlist secondary to tumor progression (11).

The most common bridging therapy options we use are transarterial chemoembolization (TACE) and percutaneous or laparoscopic ablation (11). Reported rates of complete tumor necrosis, or complete pathologic response, vary based on treatment modality with response rates between 0 (12) and 57\% (13) reported for TACE and 8.3 (12) and 74\% (14) reported for radiofrequency ablation. The primary objective of this study was to determine the rate of complete tumor necrosis after locoregional bridging therapy for HCC prior to liver transplantation, as determined by explant histopathology.

\section{MATERIALS AND METHODS}

After Institutional Review Board approval, the records of all adult patients who underwent liver transplantation between January 1, 2008 and July 31, 2015 were reviewed using the electronic medical record and a prospectively maintained transplantation database. Demographic variables were collected, including type of transplant, age, gender, calculated Model for End-stage Liver Disease (MELD) score, body mass index (BMI), etiology of disease, and medical comorbidities. MELD score was calculated using the following formula: $10 \times\{[0.957 \times \ln ($ creatinine $)]+[0.378 \times$ $\ln$ (bilirubin) $]+[1.12 \times \ln ($ INR $)]\}+6.43$, and exception points were not added. For any value $<1.0$, the value was set to 1.0 to avoid negative results. Any patient who had received dialysis twice in the previous 7 days had creatinine set to 4.0.

All patients in this cohort had a cancer therapy plan discussed at our center's multidisciplinary liver tumor board. In general, ablation is considered for patients with three or less tumors with size $<4 \mathrm{~cm}$ for any single tumor. Patients treated with at least one TACE prior to transplant were given either a tripledrug cocktail (mitomycin C, cisplatin, and doxorubicin) with Lipiodol or DC beads ${ }^{\circledR}$. TACE procedures were performed by our interventional radiology team using percutaneous femoral access and fluoroscopic guidance. Ablations were either performed laparoscopically or percutaneously using CT-guidance. Laparoscopic ablations were performed by either of two surgical oncologists using a microwave source. CT-guided ablations were performed using radiofrequency ablation or microwave ablation and were performed by an interventional radiologist. The majority of patients were treated with appropriate antiviral therapy until the time of transplant.

All liver explants were examined by a pathologist specializing in hepatobiliary disease. Tumor viability was determined using standard hematoxylin and eosin staining. The presence and extent of tumor necrosis was characterized based on an estimated percent of necrosis. Cases where there was doubt about complete response were placed in the viable tumor category for the purpose of analysis. Statistical analysis was performed using Statistical Package for the Social Sciences version 22 (IBM, Armonk, NY, USA). Categorical variables were compared using Fisher's exact test, and continuous variables were compared using the independent sample $T$-test. Survival curves were computed with the Kaplan-Meier method and differences in outcome determined by log-rank test.

\section{RESULTS}

There were 178-liver transplants completed during the study period, including 9-combined liver-kidney transplants (Table 1). The most common etiologies leading to transplantation were cirrhosis related to hepatitis $\mathrm{C}$ virus ( $\mathrm{HCV}$ ) or hepatitis $\mathrm{B}$ virus (HBV) infection, alcohol abuse, and non-alcoholic steatohepatitis. Sixty-three (35\%) patients had HCC. Fourteen (22\%) of these were diagnosed incidentally on explant pathology. Patients transplanted with HCC were older, with lower natural MELD and higher proportion of HCV and HBV infections, and more often had a history of prior abdominal surgery. Forty-three $(68 \%)$ of the 63 patients with HCC were treated with at least one bridging therapy prior to transplantation, and all patients were within Milan criteria (and none were downstaged). Eighteen (42\%) of these patients underwent TACE initially and 25 (58\%) underwent ablation. Eleven patients underwent a second bridging procedure - 10 TACE and 1 laparoscopic microwave ablation.

Patient and tumor characteristics are compared between bridging therapy groups in Table 2. Most patients had magnetic resonance imaging (MRI) at baseline prior to bridging therapy. Patients who underwent TACE had more and larger tumors. After bridging therapy, $88 \%$ of patients had follow-up imaging prior to transplantation with MRI or CT. Twenty (80\%) patients who underwent ablation and $9(60 \%)$ patients who underwent TACE had complete response to locoregional therapy based on mRECIST imaging criteria, which defines complete response as "Disappearance of any intratumoral arterial enhancement in all target lesions" (1).

The mean number of days between initial bridging therapy and transplantation was 242 (212 days for TACE patients and 249 days for ablation patients). Explant pathology characteristics are detailed in Table 2. Viable tumor was identified in explant pathology in 32 patients (74\%), including 11 (61\%) patients who underwent TACE and 21 (84\%) patients who underwent ablation. For the 21 patients who underwent ablation and had viable tumor on explant pathology, $16(76.2 \%)$ had viable tumor in the ablated nodule(s) and 5 (23.8\%) had viable tumor in both the ablated nodule(s) and in one or more separate nodule(s).

Median follow-up was 30 months for the entire cohort (33 months for patients alive at last follow-up and 16 months for patients who have died). There was no significant difference in overall survival between patients with and without HCC ( $p=0.263$, Figure 1). Survival was equivalent in patients who underwent TACE versus those who underwent ablation ( $p=0.575$, Figure 2 ). The presence or absence of viable tumor was not associated with overall survival in the HCC cohort (Figure 3); there was one patient who experienced a recurrence during the study period. No other differences in outcomes were detected between the viable and non-viable tumor groups.

The patient who experienced recurrence was a 50-year-old male with history of abdominal gunshot wound and cirrhosis secondary to HCV and HCC. He was diagnosed with two HCC 
TABLE 1 | Descriptive characteristics of entire cohort, HCC group, and non-HCC group.

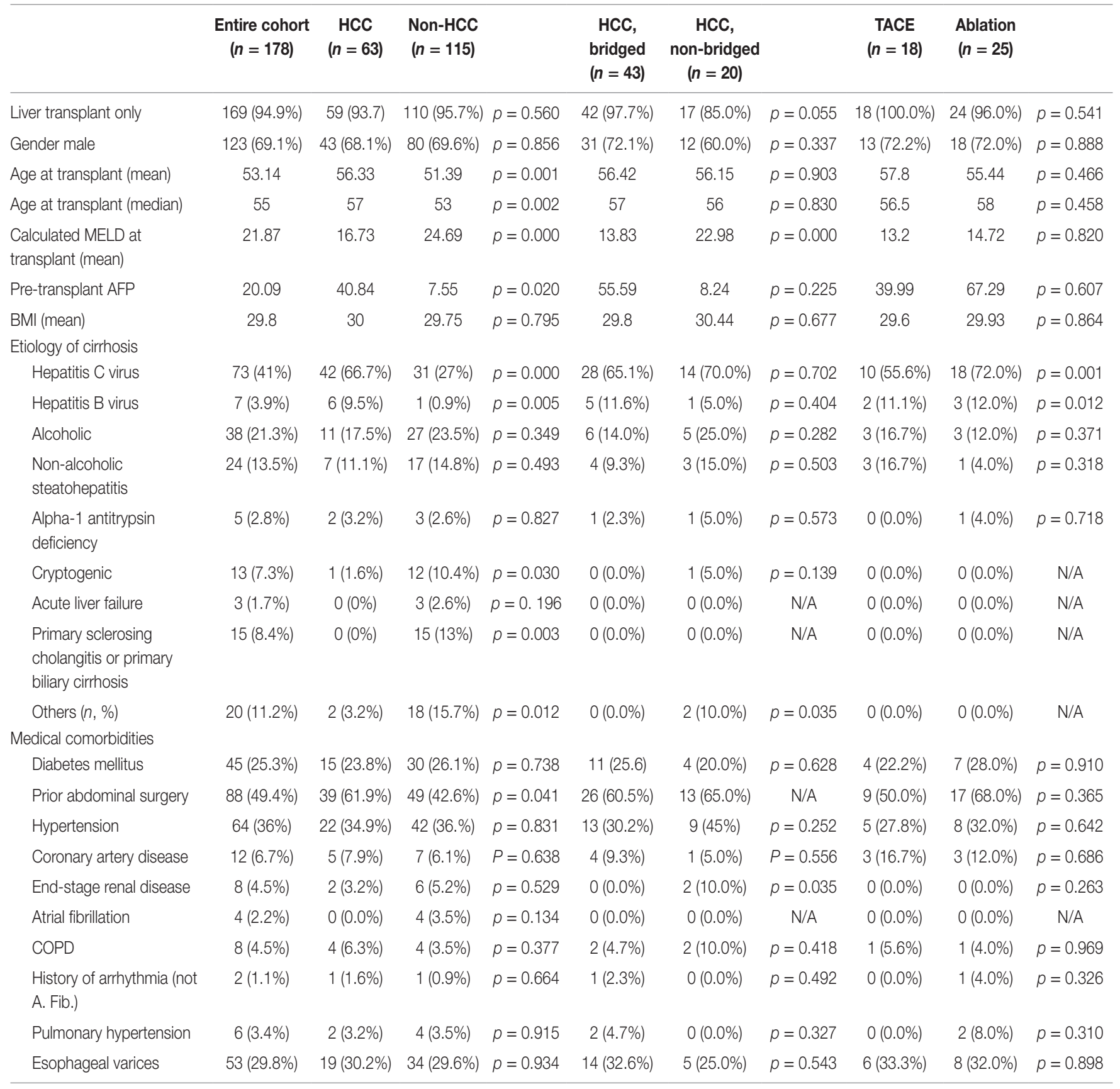

lesions in segment VI of the liver measuring $1.9 \mathrm{~cm} \times 2.1 \mathrm{~cm}$ and $1.5 \mathrm{~cm} \times 1.7 \mathrm{~cm}$. He underwent CT-guided radiofrequency ablation of both lesions. Six months later, MRI demonstrated a lesion in the left hepatic lobe concerning for HCC and the patient underwent left hepatic artery TACE. He did not undergo follow-up imaging, and 1 month later, he underwent liver transplantation. He had an uneventful postoperative course. One year after transplantation, he presented with elevated liver enzymes and underwent blind follow-up liver biopsy, which demonstrated recurrent HCC. He subsequently underwent MRI, which demonstrated multifocal tumor in the liver most consistent with HCC, osseous lesions concerning for metastases, and extensive upper abdominal and retroperitoneal lymphadenopathy. The patient and his family opted for hospice care, and he passed away prior to his next follow-up appointment.

\section{DISCUSSION}

Rates of viable tumor in the hepatic explant were high in this series after bridging therapy with either TACE or ablation. The 
TABLE 2 | Imaging and explant characteristics of patients with hepatocellular carcinoma.

\begin{tabular}{|c|c|c|c|c|c|}
\hline & & $\begin{array}{l}\text { All patients with } \\
\text { HCC }(n=63)\end{array}$ & TACE $(n=18)$ & Ablation & \\
\hline \multicolumn{6}{|l|}{ Imaging prior to bridging therapy } \\
\hline \multirow[t]{2}{*}{ Imaging type } & $\mathrm{MRI}$ & 37 (86.0\%) & 15 (83.3\%) & 21 (84.0\%) & $p=0.823$ \\
\hline & CT & $4(9.3 \%)$ & $2(11.1 \%)$ & $2(8.0 \%)$ & \\
\hline \multicolumn{2}{|l|}{ Number tumors median } & 1.5 & 1 & 1 & $p=0.014$ \\
\hline \multicolumn{2}{|l|}{ Largest tumor dimension median (cm) } & 2.7 & 2.9 & 2.3 & $p=0.149$ \\
\hline \multicolumn{6}{|l|}{ Imaging after bridging therapy } \\
\hline \multirow[t]{2}{*}{ Imaging type } & $\mathrm{MRI}$ & 37 (86.0\%) & $13(76.5 \%)$ & 23 (95.8\%) & $p=0.134$ \\
\hline & CT & $1(2.3 \%)$ & $1(5.9 \%)$ & $0(0.0 \%)$ & \\
\hline \multirow[t]{2}{*}{ Post-bridge imaging with $\mathrm{HCC}$} & Yes & $9(23.7 \%)$ & $6(40.0 \%)$ & $3(13.0 \%)$ & $p=0.104$ \\
\hline & No & $29(67.4 \%)$ & $9(60.0 \%)$ & $20(87.0 \%)$ & \\
\hline \multicolumn{6}{|c|}{ Explant - patients grouped on first bridging procedure } \\
\hline \multirow[t]{2}{*}{ Viable tumor on explant } & Yes & $50(79.4 \%)$ & $11(61.1 \%)$ & $21(84.0 \%)$ & $p=0.069$ \\
\hline & No & $13(20.6 \%)$ & $7(38.9 \%)$ & $4(16.0 \%)$ & \\
\hline \multirow[t]{5}{*}{ Number of tumors on explant pathology } & 0 & $3(4.8 \%)$ & $1(5.6 \%)$ & $1(4.0 \%)$ & $p=0.642$ \\
\hline & 1 & $32(50.8 \%)$ & $6(33.3 \%)$ & 15 (60.0\%) & \\
\hline & 2 & $13(20.6 \%)$ & $6(33.3 \%)$ & $5(40.0 \%)$ & \\
\hline & 3 & $13(20.6 \%)$ & $4(22.2 \%)$ & $3(12.0 \%)$ & \\
\hline & 4 & $2(3.2 \%)$ & $1(5.6 \%)$ & $1(4.0 \%)$ & \\
\hline \multirow[t]{2}{*}{ Explant tumor necrosis } & Yes & $39(62.9 \%)$ & $12(66.7 \%)$ & $20(80.0 \%)$ & $p=0.482$ \\
\hline & No & $23(37.1 \%)$ & $6(33.3 \%)$ & $5(20.0 \%)$ & \\
\hline \multicolumn{2}{|l|}{ Explant tumor necrosis, mean (SD) } & $34.39 \%$ & $41.20 \%$ & $47.00 \%$ & $p=0.787$ \\
\hline \multirow[t]{5}{*}{ Explant pathology T-stage } & $\mathrm{TO}$ & $3(4.8 \%)$ & $2(11.1 \%)$ & $1(4.0 \%)$ & $p=0.198$ \\
\hline & $\mathrm{T} 1$ & 27 (42.9\%) & $4(22.2 \%)$ & 13 (52.0\%) & \\
\hline & $\mathrm{T} 2$ & 23 (36.5\%) & $8(44.4 \%)$ & $6(24.0 \%)$ & \\
\hline & Т3a & $2(3.2 \%)$ & $0(0.00 \%)$ & $2(8.0 \%)$ & \\
\hline & None & $8(12.7 \%)$ & $4(22.2 \%)$ & $3(12.0 \%)$ & \\
\hline \multirow[t]{3}{*}{ Tumor grade } & 1 & 12 (24.5\%) & 2 (20.0\%) & $1(4.8 \%)$ & $p=0.346$ \\
\hline & 2 & 26 (53.1\%) & $6(60.0 \%)$ & 13 (61.9\%) & \\
\hline & 3 & $9(18.4 \%)$ & $2(20.0 \%)$ & $5(23.8 \%)$ & \\
\hline \multicolumn{2}{|c|}{ Explant largest tumor diameter in cm, mean (SD) } & 2.8 & 2.4 & 3.8 & $p=0.141$ \\
\hline
\end{tabular}

presence of viable tumor in the hepatic explant was not associated with worse outcome, although our numbers may be underpowered to detect a difference. Agopian et al. recently reported a large series of 501 patients who underwent locoregional therapies prior to liver transplantation at a single high-volume institution and concluded that 126 patients (25\%) had complete tumor necrosis. Contrary to our results, they found that complete tumor necrosis was associated with superior outcomes. Overall survival was not reported in this study. Transplant was done for $13 \%$ of patients originally outside of Milan criteria in this series, and the overall recurrence of HCC was $15.0 \%$ in patients without complete pathologic response and $2.4 \%$ in patients with complete pathologic response (15). While the rate of recurrence in our study was lower, patient selection is the likely explanation as all patients transplanted had HCC within Milan criteria (and none were downstaged).

TACE and ablation are common bridging therapies for HCC prior to transplantation (15). Reported rates of complete tumor necrosis, or complete pathologic response, vary based on treatment modality with response rates between 0 (12) and 57\% (13) reported for TACE and 8.3 (12) and 74\% (14) reported for radiofrequency ablation. Generally, patients who achieve transplant after these bridging therapies have excellent outcomes with good overall and recurrence-free survival, and these therapies are well tolerated without high risk of morbidity $(16,17)$. Guidelines recommend that patients with HCC be considered for bridging therapy (10) if they are expected to wait more than 6 months for transplantation in order to reduce dropouts from the waitlist secondary to tumor progression (11).

This is consistent with prior studies. Marin et al. evaluated 28 patients with 38 nodules who underwent TACE, selective internal radiation therapy (SIRT), and chemical or radiofrequency ablation and noted that $42 \%$ of patients had post-bridging imaging concerning for HCC and that there was viable tumor in 35 (90\%) of the 39 treated nodules on explant pathology (12). Lu et al. looked at 47 HCC nodules in 24 patients treated with single or double RFA prior to liver transplantation and concluded that 35 (74\%) of the tumors were successfully treated. They determined that small lesions and non-perivascular lesions were more likely to be successfully treated with this modality and concluded that RFA is an effective treatment for small $(<3 \mathrm{~cm})$ HCC (14). Wong et al. evaluated patients who had undergone TACE, percutaneous 


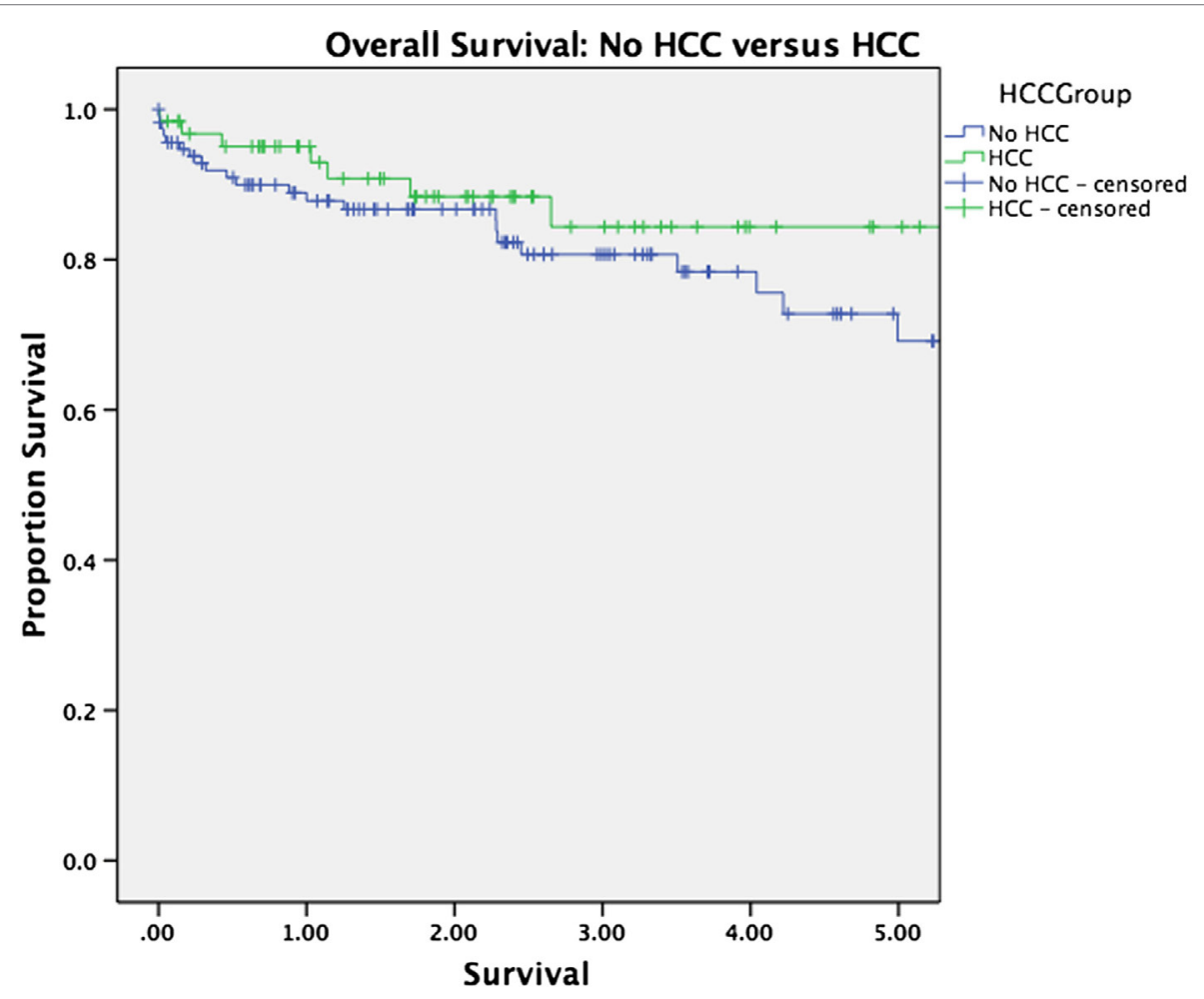

FIGURE 1 | Kaplan-Meier survival curves are shown for patients transplanted with and without HCC

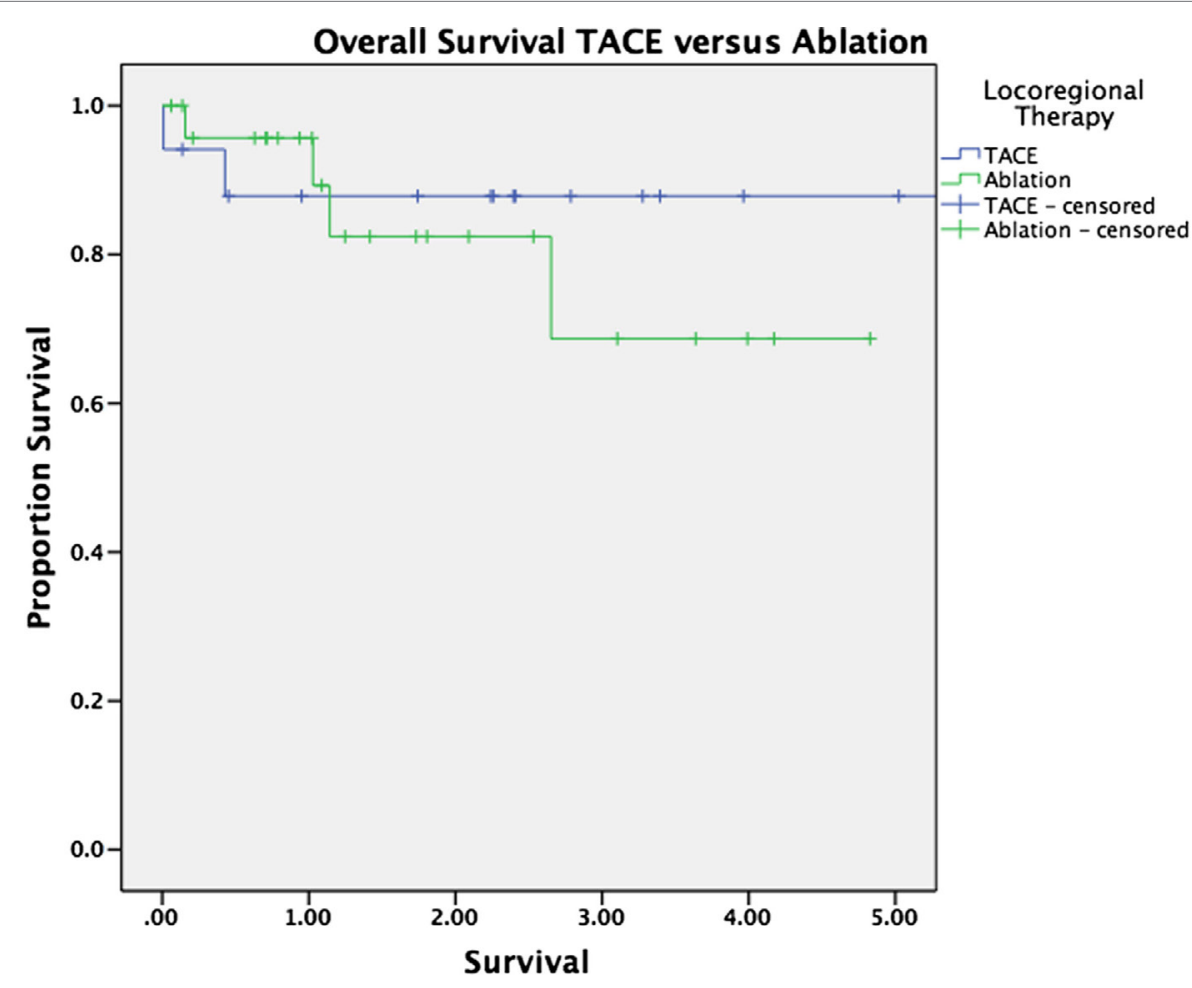

FIGURE 2 | Kaplan-Meier survival curves are shown for patients bridged with TACE and those bridged with ablation. 


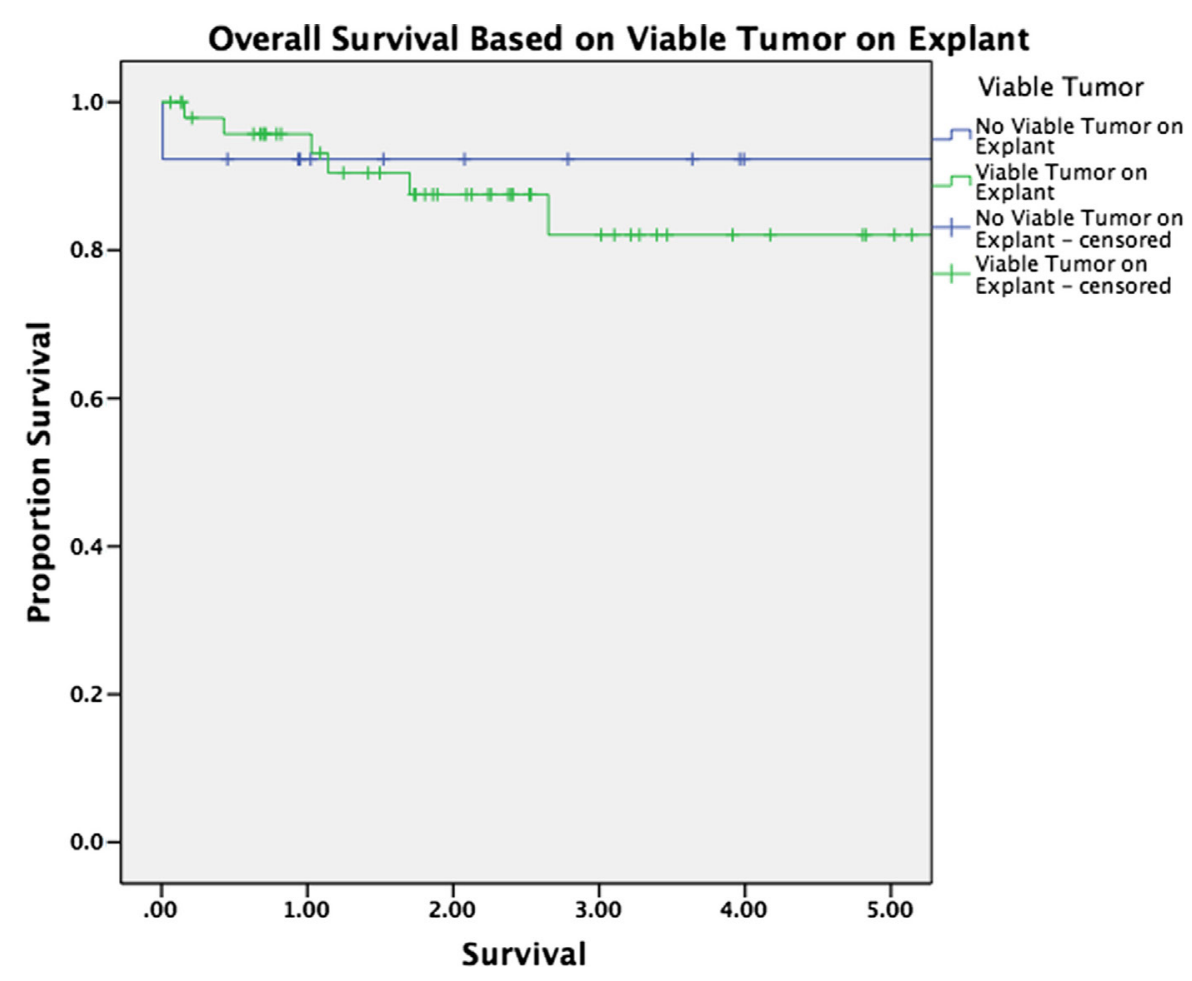

FIGURE 3 | Kaplan-Meier survival curves are shown for patients with viable tumor on explant pathology and without viable tumor on explant pathology after bridging therapy.

ethanol injection (PEI), and radiofrequency ablation (RFA) and determined that of 44 treated nodules, 29 (66\%) had a least $75 \%$ necrosis (18).

Limitations of this study include retrospective design, small sample size, and median follow-up slightly $<3$ years. As mentioned, this may mean that the analysis lacked power to detect an influence of tumor viability on outcome. Additionally, due to the small size of our cohort, we were not able to detect differences in other outcomes, including the quality of life. Since the number of recurrence cases of HCC is very low, we conclude that transplant after bridging therapy for patients within Milan criteria does not require serial bridging to achieve complete pathologic tumor response. The presence of complete response by imaging is adequate to allow close observation with serial imaging prior to transplant.

In conclusion, we determined that although complete pathologic response to bridging therapy is not common, this does not have an impact on transplantation outcomes. Alternate methods

\section{REFERENCES}

1. Lencioni R, Llovet JM. Modified RECIST (mRECIST) assessment for hepatocellular carcinoma. Semin Liver Dis (2010) 30:52-60. doi:10.105 5/s-0030-1247132

2. El-Serag HB, Davila JA, Petersen NJ, Mcglynn KA. The continuing increase in the incidence of hepatocellular carcinoma in the United States: an update. Ann Intern Med (2003) 139:817-23. doi:10.7326/0003-4819-13910-200311180-00009 may be required to determine if tumor cells that appear viable on pathologic analysis are actually alive. Further studies in this area could include assessing whether bridged cells are less likely to grow in culture or in xenografts in comparison to non-bridged (incidental) tumors.

\section{AUTHOR CONTRIBUTIONS}

$\mathrm{EB}, \mathrm{CS}$, and MD were responsible for initial study design. $\mathrm{EB}$ and $\mathrm{KD}$ collected data and performed the data analysis. $\mathrm{EB}, \mathrm{KD}, \mathrm{AH}$, $\mathrm{AM}, \mathrm{LC}, \mathrm{GD}, \mathrm{SB}, \mathrm{PB}, \mathrm{MD}$, and CS performed interpretation of the data, composed the manuscript, and provided critical review and revision.

\section{FUNDING}

No funding was received for this study. EB would like to acknowledge her T32 support, NIH T32AI 106704-01A1.

3. Davila JA, Morgan RO, Shaib Y, Mcglynn KA, El-Serag HB. Hepatitis C infection and the increasing incidence of hepatocellular carcinoma: a population-based study. Gastroenterology (2004) 127:1372-80. doi:10.1053/j. gastro.2004.07.020

4. El-Serag HB, Marrero JA, Rudolph L, Reddy KR. Diagnosis and treatment of hepatocellular carcinoma. Gastroenterology (2008) 134:1752-63. doi:10.1053/j. gastro.2008.02.090

5. Grazi GL, Ercolani G, Pierangeli F, Del Gaudio M, Cescon M, Cavallari A, et al. Improved results of liver resection for hepatocellular carcinoma 
on cirrhosis give the procedure added value. Ann Surg (2001) 234:71-8. doi:10.1097/00000658-200107000-00011

6. Di Bisceglie AM. Issues in screening and surveillance for hepatocellular carcinoma. Gastroenterology (2004) 127:S104-7. doi:10.1053/j. gastro.2004.09.022

7. Forner A, Hessheimer AJ, Isabel Real M, Bruix J. Treatment of hepatocellular carcinoma. Crit Rev Oncol Hematol (2006) 60:89-98. doi:10.1016/j. critrevonc.2006.06.001

8. Taefi A, Abrishami A, Nasseri-Moghaddam S, Eghtesad B, Sherman M. Surgical resection versus liver transplant for patients with hepatocellular carcinoma. Cochrane Database Syst Rev (2013) 6:CD006935. doi:10.1002/14651858. CD006935.pub2

9. Llovet JM, Fuster J, Bruix J. Intention-to-treat analysis of surgical treatment for early hepatocellular carcinoma: resection versus transplantation. Hepatology (1999) 30:1434-40. doi:10.1002/hep.510300629

10. Mehta N, Dodge JL, Goel A, Roberts JP, Hirose R, Yao FY. Identification of liver transplant candidates with hepatocellular carcinoma and a very low dropout risk: implications for the current organ allocation policy. Liver Transpl (2013) 19:1343-53. doi:10.1002/lt.23753

11. Prasad MA, Kulik LM. The role of bridge therapy prior to orthotopic liver transplantation. J Natl Compr Canc Netw (2014) 12:1183-1190; quiz 1191.

12. Marin HL, Furth EE, Olthoff K, Shaked A, Soulen MC. Histopathologic outcome of neoadjuvant image-guided therapy of hepatocellular carcinoma. J Gastrointestin Liver Dis (2009) 18:169-76.

13. Pompili M, Francica G, Ponziani FR, Iezzi R, Avolio AW. Bridging and downstaging treatments for hepatocellular carcinoma in patients on the waiting list for liver transplantation. World J Gastroenterol (2013) 19:7515-30. doi:10.3748/wjg.v19.i43.7515

14. Lu DS, Yu NC, Raman SS, Limanond P, Lassman C, Murray K, et al. Radiofrequency ablation of hepatocellular carcinoma: treatment success as defined by histologic examination of the explanted liver. Radiology (2005) 234:954-60. doi:10.1148/radiol.2343040153

15. Agopian VG, Morshedi MM, Mcwilliams J, Harlander-Locke MP, Markovic D, Zarrinpar A, et al. Complete pathologic response to pretransplant locoregional therapy for hepatocellular carcinoma defines cancer cure after liver transplantation: analysis of 501 consecutively treated patients. Ann Surg (2015) 262:536-45. doi:10.1097/SLA.0000000000001384

16. Liang P, Wang Y, Yu X, Dong B. Malignant liver tumors: treatment with percutaneous microwave ablation - complications among cohort of 1136 patients. Radiology (2009) 251:933-40. doi:10.1148/radiol.2513 081740

17. Ahmed S, De Souza NN, Qiao W, Kasai M, Keem LJ, Shelat VG. Quality of life in hepatocellular carcinoma patients treated with transarterial chemoembolization. HPB Surg (2016) 2016:6120143. doi:10.1155/2016/ 6120143

18. Wong LL, Tanaka K, Lau L, Komura S. Pre-transplant treatment of hepatocellular carcinoma: assessment of tumor necrosis in explanted livers. Clin Transplant (2004) 18:227-34. doi:10.1111/j.1399-0012.2004.00164.x

Conflict of Interest Statement: The authors declare that the research was conducted in the absence of any commercial or financial relationships that could be construed as a potential conflict of interest.

Copyright (c) 2016 Beal, Dittmar, Hanje, Michaels, Conteh, Davidson, Black, Bloomston, Dillhoff and Schmidt. This is an open-access article distributed under the terms of the Creative Commons Attribution License (CC BY). The use, distribution or reproduction in other forums is permitted, provided the original author (s) or licensor are credited and that the original publication in this journal is cited, in accordance with accepted academic practice. No use, distribution or reproduction is permitted which does not comply with these terms. 